\title{
BMJ Open Exploring the frequency, duration and experience of seclusion for women in a forensic mental health setting: a mixed- methods study protocol
}

\author{
Alison Claire Hansen (D) ,, ${ }^{1,2}$ Michael Hazelton, ${ }^{1,3}$ Robyn Rosina, ${ }^{1}$ Kerry J Inder ${ }^{1,3}$
}

To cite: Hansen AC, Hazelton M, Rosina R, et al. Exploring the frequency, duration and experience of seclusion for women in a forensic mental health setting: a mixed-methods study protocol. BMJ Open 2021;11:e044261. doi:10.1136/ bmjopen-2020-044261

- Prepublication history for this paper is available online. To view these files, please visit the journal online (http://dx.doi. org/10.1136/bmjopen-2020044261).

Received 01 September 2020 Accepted 12 May 2021

Check for updates

(C) Author(s) (or their employer(s)) 2021. Re-use permitted under CC BY-NC. No commercial re-use. See rights and permissions. Published by BMJ.

${ }^{1}$ School of Nursing \& Midwifery, College of Health, Medicine and Wellbeing, University of Newcastle, Callaghan, New South Wales, Australia ${ }^{2}$ School of Nursing \& Midwifery, Faculty of Medicine, Nursing and Health Sciences, Monash University, Clayton, Victoria, Australia

${ }^{3}$ Hunter Medical Research Institute, New Lambton, New South Wales, Australia

Correspondence to Ms Alison Claire Hansen; alison.hansen@uon.edu.au

\section{ABSTRACT}

Introduction Seclusion use is high in forensic mental health settings and is associated with avoidable physical and psychological harm. The use of seclusion causes significant distress and trauma for those secluded and women in these settings are particularly vulnerable. This study protocol aims to identify factors associated with the use of seclusion and the experience of seclusion for women in forensic mental health settings.

Methods and analysis This study will use a prospective mixed-methods design. Quantitative data on the frequency and duration of seclusion and potential explanatory demographic and clinical variables will be collected prospectively from consecutive medical records of women admitted to a forensic mental health service over a 2-year period. Data will be analysed using descriptive statistics and logistic regression techniques. Qualitative data will be collected from individual face-to-face semistructured interviews with women who have experienced seclusion about the reason for seclusion, whether they agreed with the reason for seclusion, their experience of the seclusion event and the seclusion environment and support provided while in seclusion using qualitative description methods. Data will be analysed using thematic analysis.

Ethics and dissemination Ethical approval has been granted by the University of Newcastle Human Research Ethics Committee (H-2019-0122) and organisational support granted from the participating forensic mental health service. Participation will be voluntary and written informed consent is required. Results will be disseminated via the first author's doctoral thesis, in peerreviewed journals and at conferences. Results will inform recommendations for policy, and evidence for timely and specific interventions to support the reduction of seclusion for women in forensic mental health settings.

\section{INTRODUCTION}

Seclusion can be defined as 'the confinement of a patient at any time of the day or night alone in a room or area from which free exit is prevented'. ${ }^{1}$ Seclusion is used as a behavioural intervention to protect the person or others from harm, however its use is associated with significant risk for physical and psychological harm to those who experience seclusion. ${ }^{2}$ Despite the risks, seclusion continues to be
Strengths and limitations of this study

- This study uniquely explores the frequency, duration and experience of seclusion specifically for women in a forensic mental health setting prospectively.

- The mixed-methods design allows the consumer voice to be expressed and acknowledged, supporting the notion of recovery in the mental health context.

- The potential limited sample may present challenges with generalisability due to the small number of women in forensic mental health settings and the inability to recruit women whom one researcher has previously worked with in the clinical environment.

used without evidence supporting its effectiveness or benefits for treatment. ${ }^{3}$ Reducing, and where possible eliminating the use of restrictive interventions such as seclusion, has been a priority for health services, following reports of injury and death resulting from its use. ${ }^{4}$ Strategies aimed at reducing seclusion use appear to have a positive effect. ${ }^{5}$ However, seclusion use and the frequency and duration of a seclusion event, differ considerably between settings ${ }^{67}$ and use in forensic settings remains high.

In Australia, the rates of seclusion in forensic mental health settings are higher than in general settings. The Australian Institute of Health and Welfare reported 21.2 seclusion events per 1000 bed days in forensic settings for the period 2018-2019, compared with 7.5 seclusion events per 1000 bed days for general mental health settings. ${ }^{1}$ Forensic mental health settings have seen an increase in seclusion events from the period 2014-2015 to 2018-2019, with an average annual change of $31.5 \% .^{1}$ The duration of seclusion events is also higher in forensic settings compared with general mental health settings (48.1 hours and 4.5 hours, respectively). ${ }^{1}$ While the duration is higher, forensic settings have seen a 
greater overall decrease compared with general settings in the duration of seclusion from the period 2014-2015 to $2018-2019: 11.4 \%$ compared with $8.6 \%$, respectively. ${ }^{1}$ The reason for the differences between seclusion rates for forensic mental health settings and general mental health settings are unclear, however, patient demographics, presentation complexity, seclusion use, culture and service delivery models may account for variances in seclusion rates across services. ${ }^{8}$

Males and females admitted to forensic mental health settings are comparable in relation to their characteristics, clinical presentation and history of mental health problems. ${ }^{9}$ However, sex differences have been identified in diagnosis and incidents of self-harm or violence during admission, which may necessitate the need for seclusion to manage behaviour where other interventions have been unsuccessful. Major depression with psychosis, and borderline personality disorder are diagnosed more frequently in women than men. ${ }^{9}$ Women engage in more self-harm ${ }^{10}$ and are involved in more frequent incidents of violence ${ }^{1011}$ while admitted to forensic mental health settings, compared with men. Self-harm and harm directed at others may be an indication of psychological disturbance and a way to express distress and torment that women have experienced. ${ }^{11}$ Previous studies have found that borderline personality traits and threats of self-harm which are more prevalent in women, may contribute to higher rates of seclusion. ${ }^{12}$ Women in forensic mental health settings have often experienced extensive trauma, domestic violence and abuse throughout their lives, ${ }^{13-15}$ which may lead to or exacerbate the psychological disturbance experienced when secluded.

Seclusion can result in physical and psychological harm ${ }^{2}$ and the negative effects of seclusion may persist even after seclusion has ended. ${ }^{16}$ While seclusion continues to be used in forensic mental health settings, understanding what factors are associated with women being secluded and the experience of seclusion, may provide support in reducing seclusion and harm associated with its use in this setting. To the authors' knowledge, there has been no study which examines both the use of seclusion and the experience of seclusion for women in forensic mental health settings.

\section{AIMS}

This study aims to identify factors associated with the frequency and duration of seclusion events for women in forensic mental health settings, and to explore the experiences of women who are subjected to seclusion in forensic mental health settings.

\section{METHODS AND ANALYSIS}

\section{Study design}

The study will achieve the study aims by using a concurrent mixed-methods study design ${ }^{17}$ with quantitative and qualitative data collected simultaneously. A mixed-methods study design allows the researcher to collect both quantitative and qualitative data and give equal emphasis to both types of data. ${ }^{17} \mathrm{~A}$ mixed-methods approach was chosen to gain perspectives from the two different types of data and will provide study participants a voice, which will support the findings being grounded in the participants' experiences. Quantitative data will be collected prospectively in component one using a cohort study design with demographic, clinical and seclusion data being collected on consecutive women admitted. Qualitative data will be collected in component two using qualitative description ${ }^{18}$ whereby women who experience seclusion will be interviewed to explore and understand their experience of seclusion.

\section{Patient involvement}

Patients were not involved in the design, conduct, or reporting of the study. The authors have actively engaged with the organisation's research committee in refining the study design where appropriate, and with hospital staff who work closely with and who are advocates for the patients. Where requested, results will be provided to all participants.

\section{Study setting}

The study will be conducted at an acute female unit within a high secure forensic hospital in Australia. Forensic mental health has been broadly defined as 'an area of specialisation that, in the criminal sphere, involves the assessment and treatment of those who are both mentally disordered and whose behaviour has led, or could lead to, offending' (19 p 307). Forensic hospitals provide a safe and secure environment in order for assessment and treatment to occur, for those with a mental health disorder and have, or may be, at risk of offending.

\section{Study participants}

The study participants will include women aged 18 years and over admitted to the study site during the 24-month study period, estimated to be 95 women. Inclusion criteria will be consenting female patients, aged 18 years and over. Women who do not consent or do not have the capacity to consent or do not speak or understand written and verbal English will be excluded from participating in the study.

\section{Sampling and sample population}

The study population for the cohort study includes all consecutive women admitted to the study site during the study time frame. The study population for the qualitative descriptive study includes women admitted to the study site who have been secluded during the study time frame. Using purposive sampling women who experience seclusion will be invited to participate in a semistructured interview to discuss their experience of seclusion at not less than 24 hours following the cessation of the seclusion event. Where a woman has been secluded more than once during the study time frame and during their current admission, the woman will be invited to participate in 
an interview after each seclusion event. Women deemed too acutely unwell or too high risk, will be excluded from participating in the qualitative component. It is anticipated that $10-15$ women will be recruited, with a saturation of themes being reached at this number.

\section{Recruitment of study participants}

Participants will be recruited on admission to the female acute unit, within the forensic mental health setting by the first author. The first author will contact the unit on a weekly basis to ascertain whether there has been a patient admission, or a seclusion event. When there has been an admission and/or seclusion event, study recruitment will commence.

Prior to recruitment occurring for either component, women will be assessed by a clinician involved in the delivery of care to determine suitability to be approached by the researcher. This assessment involves the clinician completing a brief two-item survey designed to indicate whether the woman has been behaviourally settled over the past 24 hours and to ascertain the level of acuity of symptoms which may affect the ability of the first author to determine the woman's capacity to consent.

Women will not be approached to participate in the cohort study until 7 days following their admission, to allow time for the woman to adjust to being admitted to the unit. Women will not be approached to participate in an interview until 24 hours after their seclusion event has ceased, to reduce any distress that may arise as a result of being recruited to discuss their experience of seclusion. Where a woman experiences seclusion on more than one occasion during a single admission, the woman will be approached to participate in the study after each seclusion event.

Where a woman is found to be suitable to be approached by the first author, the first author will assess eligibility for recruitment and invite the woman to participate if eligible. In the event where a woman is determined not to be eligible to be approached for recruitment, suitability can be reassessed at a later date for the cohort study, or within 14 days for the qualitative study (to reduce the likelihood of recall bias). Recruitment to participate in the study requires informed consent which will be assessed by the first author who has a background in forensic mental health nursing practice and undergraduate and postgraduate education.

Following the woman being provided verbal and written information about either the cohort study or the qualitative descriptive study, there will be three possible outcomes for the recruitment process. If it is determined that the woman has capacity to consent and agrees to participate, this will be indicated on the participant consent form. Second, if it is determined that the woman does not have capacity to consent, recruitment ends. At this stage for the cohort study, the woman can be reapproached to be recruited at another time during their admission as the collection of quantitative data relies on demographic and clinical information which is unlikely to change and is collected directly from medical records. For the qualitative study, if it is determined the woman does not have capacity, capacity can be reassessed within 14 days of the cessation of the seclusion event, to reduce recall bias. The third outcome of the recruitment process
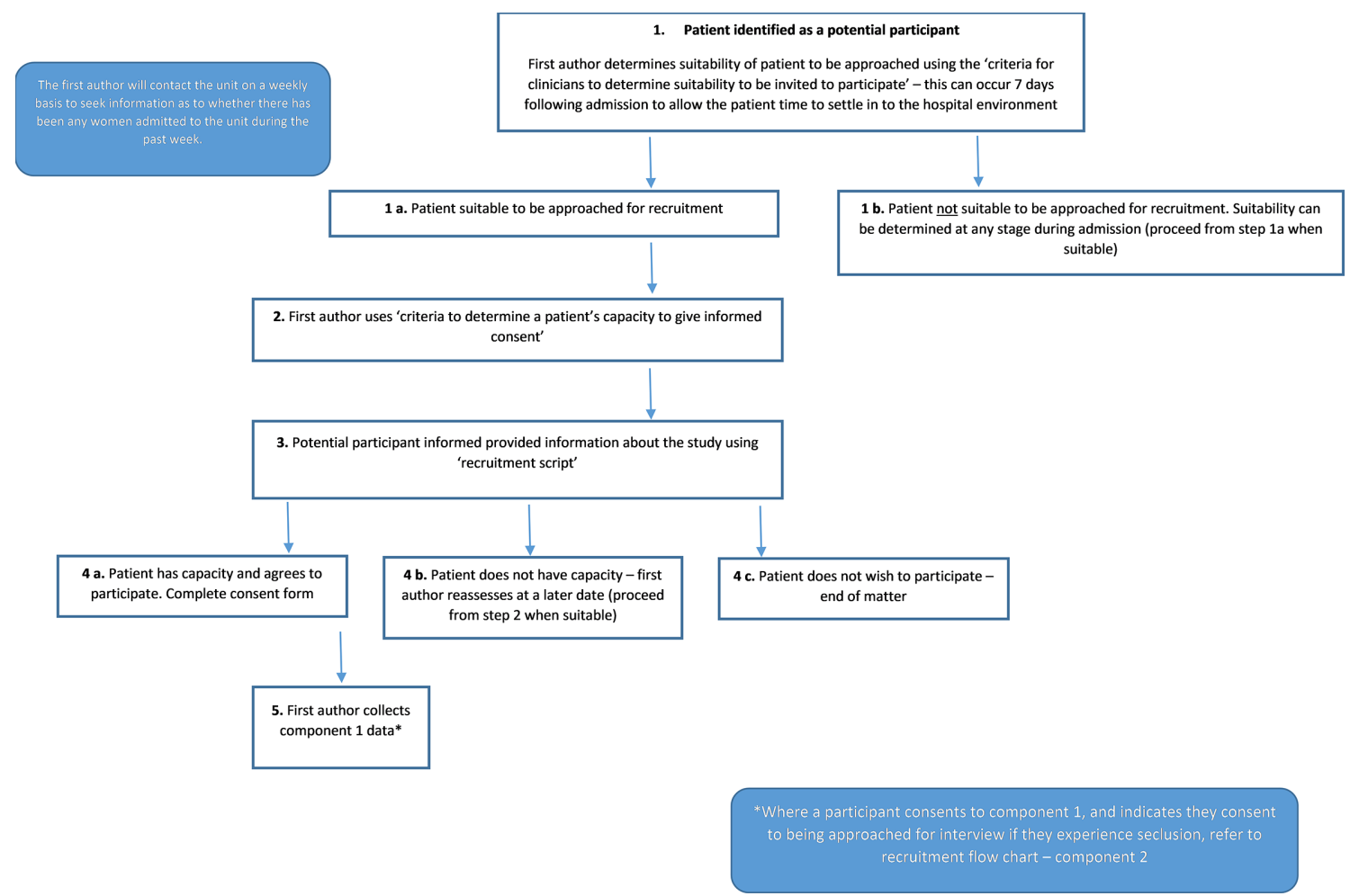

Figure 1 Recruitment process for component 1 (cohort study). 


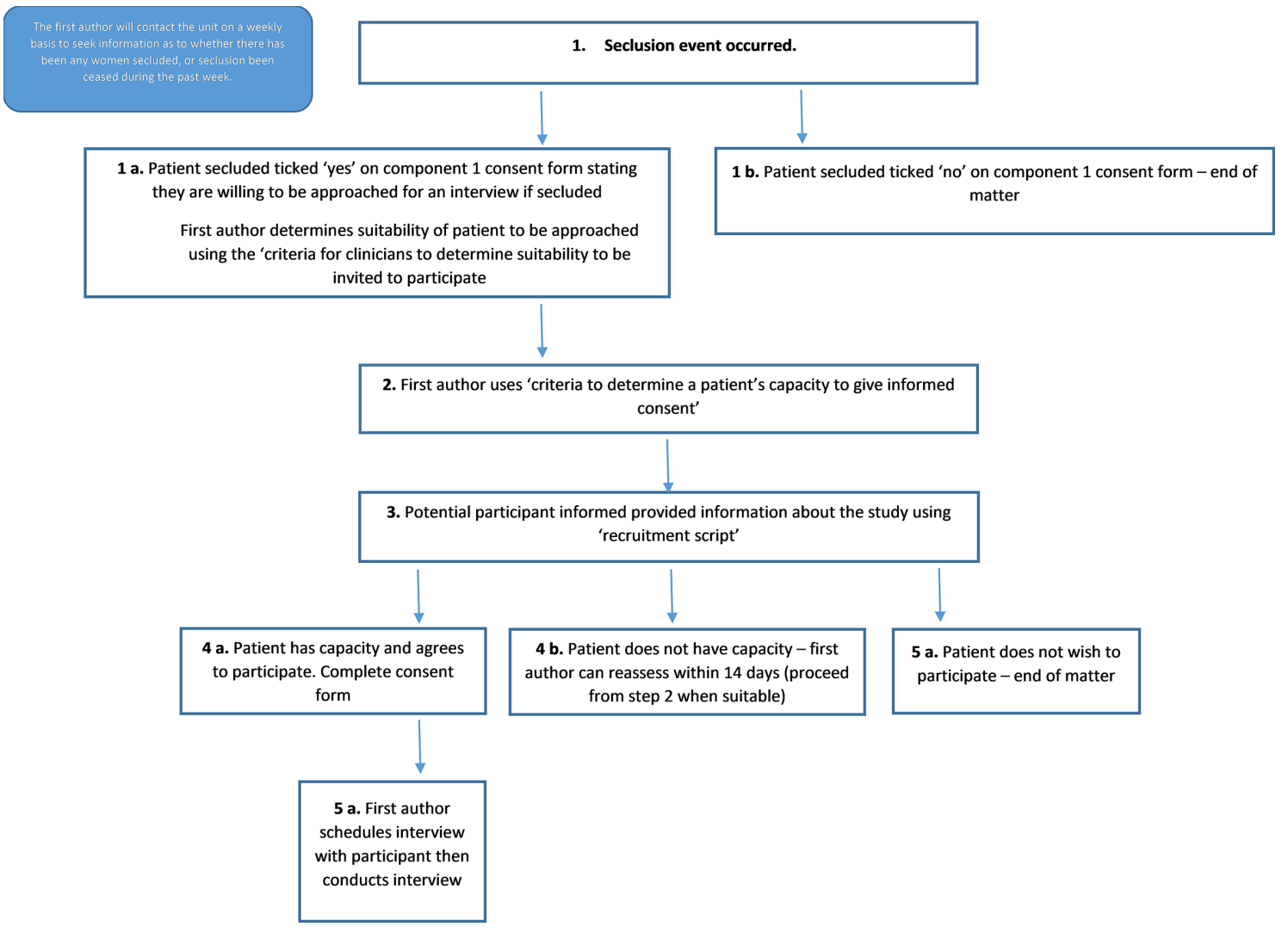

Figure 2 Recruitment process for component 2 (qualitative study).

may occur when the woman may or may not have capacity to consent, but does not wish to participate.

The recruitment process for components one and two is summarised in figures 1 and 2.

\section{Data sources}

The quantitative component will collect the participants' demographic and clinical information from the participant's medical records. Data relating to seclusion such as the date seclusion commenced and ceased, duration in hours and the documented reason for seclusion will be collected if secluded. For participants who experience seclusion more than once, the frequency will be recorded. Data will be recorded directly intoResearch Electronic Data Capture, a secure web application for building and managing online surveys and databases ${ }^{20} 21$ and stored securely on the managing research institute's server.

The qualitative component of the study will involve semi-structured interviews with women who have been secluded. The interview will consist of questions relating to the woman's experience of seclusion and will seek to explore the woman's understanding of the reason for seclusion, whether they agree with the reason for seclusion, the experience of seclusion (including the physical environment) and the support provided by the healthcare team while in seclusion. Where a woman has been secluded more than once since the researcher's last contact with the unit and agrees to participate in an interview, the interview can include both seclusion events with separation of data for each event. Other data collected will be the woman's age, date and duration of the seclusion event and reason for seclusion. Interviews will be audio recorded for later transcription, with the digital files stored on a password protected device. Following transcription, the digital files and transcript will be checked for accuracy, then the digital file destroyed. Pseudonyms will be used to protect the participants' identity.

Where a participant participates in both component one and two, consent will be sought to link this data. An additional file will be held separately to the data files which will contain an alphanumeric participant identification code linked to the participant's name and date of birth to allow data to be reidentified for the purpose of linking component one data to component two data only.

\section{Power and data analysis}

Sample size estimation for the cohort study is based on estimated admission rates to the study site, where seclusion events are estimated to be 11-12 events per 1000 bed days over a 2-year data collection period. This will enable estimation of the true rate of seclusion events with a $20 \%$ margin of error for a $95 \%$ CI. This sample is anticipated to result in an estimated 50 seclusion events over 2 years (which may include participants with more than one seclusion event), with an estimated $20 \%$ non-consent rate. Using the rule of thumb of 5-10 events per variable, ${ }^{22}$ there should be sufficient data to estimate five regression coefficients without risk of overfitting the model. For the qualitative study, interviews with 10-15 participants are 
anticipated to result in an appropriate sample size, ${ }^{23} 24$ where saturation of themes are achieved.

Quantitative data will be checked for implausible values, errors and missing data and summarised descriptively. Continuous data will be assessed for normality by comparing the mean and median values and constructing histograms and summarised as a mean and standard deviation (or median and IQR). Cross-tabulations will be used to examine the relationship between two or more categorical variables. Data will initially be analysed using simple logistic regression techniques for the frequency of seclusion, categorised as a binary outcome of no seclusion events vs at least one seclusion event and will include the entire sample of women. Duration of seclusion (in minutes), will be analysed as a continuous variable using linear regression techniques if there is a sufficient sample and if the data are normally distributed. Otherwise data will be dichotomised and analysed using logistic regression. The analysis of factors associated with duration of seclusion will be undertaken only on those participants who experienced seclusion.

Variables for inclusion in these analyses will include age, marital status (married or defacto/divorced or separated/ single/widowed), dependents (yes, how many? And are they normally in the woman's care?/no), level of education (primary school/high school/Technical and Further Education or Vocation/tertiary), employment status prior to admission (employed/unemployed/disability support pension/student/never employed/other), Aboriginal or Torres Strait Islander background (yes/no), country of origin (Australia/New Zealand/other), primary reason for admission, mental health history, previous admissions to a forensic mental health setting, past history of trauma (yes/no), experienced seclusion (yes/no), reason for seclusion and current medications related to mental health and/or physical health. Demographic data and clinical characteristics will be collected to account for potential confounding. As the study is non-randomised, non-identified potential confounding variables will not be accounted for. Quantitative data will be analysed using statistical software SPSS (IBM) or STATA (StataCorp).

For women who do not consent to component one, the person's age in years and admission diagnosis will be recorded to allow a comparison between consenters and non-consenters. This will allow estimation of potential recruitment bias.

Qualitative data will be interpreted through a thematic analysis methodology which will allow for the identification and analysis of themes within qualitative data. ${ }^{25}$ The process of thematic analysis includes six phases of analysis; familiarisation with the data, the generation of codes, search for and review of themes, definition of the themes and production of the report. ${ }^{25}$ The familiarisation phase will involve immersion in the data through reading interview transcripts, listening to audio transcripts and making notes; generating codes will allow for the identification and labelling of data features relevant to the research question; searching for themes will develop from the generation of codes and captures patterned responses or meaning in the data; reviewing the themes allows for quality checking through comparison of themes and data extracts; defining themes will allow for a clear description of the theme (and subthemes if relevant) and the final phase will occur through the production of a report and dissemination. ${ }^{25}$ Following this process allows for maximisation of trustworthiness and rigour. ${ }^{26}$ Qualitative data will be analysed using NVivo (QSR International).

\section{DISCUSSION}

Reducing the use of restrictive interventions such as seclusion, is a global priority. Strategies aimed at reducing seclusion use have been successful. ${ }^{5}$ However, seclusion use in forensic mental health settings is high, and there is a lack of research that considers the use and experience of seclusion for women in this setting. ${ }^{27}$

The study aims will be achieved through prospectively collecting and examining demographic and clinical data of all consenting women admitted to the study setting, including women who do experience seclusion and those who do not over a 2-year period. Specific data about seclusion will be collected for those women who experience seclusion.

This study will allow for factors that are associated with the frequency and duration of seclusion for women in forensic mental health settings to be identified. This study will also provide an understanding of women's experiences of seclusion and identify potential links to the frequency and duration of seclusion events. Understanding the factors associated with the use of seclusion for women in forensic mental health settings will allow for the development of specific and timely interventions in order to reduce the use of seclusion and reduce the psychological distress associated with the use of seclusion. Future research that explores clinical staff experiences and their views of seclusion use for women in this setting would support the development and implementation of evidence-based interventions to reduce seclusion.

\section{Ethics and dissemination}

The study has received ethical approval from the University of Newcastle Human Research Ethics Committee (H-2019-0122) and organisational support from the participating forensic mental health service. The organisation supporting this study has not requested approval from the Aboriginal Health and Medical Research Council due to the small number of women admitted to hospital. However, the Ethical conduct in research with Aboriginal and Torres Strait Islander Peoples and communities: Guidelines for researchers and stakeholders ${ }^{28}$ has been consulted in the development of this research project. Aboriginal and Torres Strait Islander cultural values have been considered in the design and conduct of this research.

This paper reports protocol V.1 and has been written with reference to the Strengthening the Reporting 
of Observational Studies in Epidemiology (STROBE) guidelines ${ }^{29}$ and the Consolidated Criteria for Reporting Qualitative Research (COREQ) ${ }^{30}$ guidelines, to promote comprehensive and quality reporting. The STROBE guidelines provide reporting recommendations for observational studies to ensure that the study plan was followed and details findings. ${ }^{29}$ The COREQ guidelines promote comprehensive reporting of the qualitative study and includes necessary components such as research team, methods, findings, analysis and interpretations. ${ }^{30}$

\section{Ethical considerations}

A number of ethical and safety concerns were addressed prior to commencement of the study relating to the vulnerability of the population and of the researcher collecting the data. The study population includes people with a mental disorder whose severity or acuity of symptoms may impact their ability to consent. The study population may also include Aboriginal and/or Torres Strait Islander people. Capacity to consent will be assessed, and participants will be offered the opportunity to discuss their participation with a person who is able to act as an advocate and support them in understanding the study. For people who identify as an Aboriginal or Torres Strait Islander person, an Indigenous health worker or support person will be offered to discuss their participation and understanding of the study.

Participation in this study will be based on voluntary written informed consent. Determination of a woman's capacity to consent to participate will consider her understanding of the research and if she is able to remember the information provided. Check questions will be utilised to ensure information is understood and can be used to make a decision and is able to communicate their decision. ${ }^{31}$ All participants will be provided with verbal and written information about the study to support them in making their decision. Participants will be informed that their personal information will not be disclosed and will be de-identified. No specific information of the service or location will be published to minimise the risk of participants being identified in dissemination of findings.

Participants who undertake an interview will be reimbursed for their time. However, where a participant experiences seclusion on more than one occasion, reimbursement for future interview/s will not occur, therefore reimbursement will only occur for the first interview they participate in. This aims to reduce the risk of participants potentially putting themselves or others at risk in order to experience seclusion and subsequently being offered to participate in an interview in order to seek reimbursement.

The nature of experiencing and then discussing the experience of seclusion may cause distress to participants. When participating in the interview, participants will be asked questions about the seclusion event, how they felt during seclusion and will be asked to describe their experience. In doing this, distress or discomfort may be felt by the participants. As the participants are a vulnerable population (persons with a mental illness and may also be Aboriginal or Torres Strait Islander peoples), participants may be more vulnerable to discomfort. To minimise the risk of distress or discomfort, participation is optional and informed consent is required. Participants will be able to withdraw their consent or terminate an interview at any time and do not need to provide an explanation. The researcher conducting the interviews will reassure the participant of confidentiality and that their participation and responses will not influence the care they receive. Participants will be able to take a short break from the interview, end the interview and reschedule if they wish. If the participant requests a short break or to reschedule the interview, this will be reported to the participant's treating doctor and/or psychiatrist, and nurse to ensure appropriate follow-up is established.

There are a number of risks to the researcher conducting the interviews (first author) that were addressed prior to commencement of the study. The researcher is at risk of discomfort or distress from listening to participant's stories or from participants becoming aggressive or distressed during the interview. This researcher will be provided with debriefing sessions within 24-48 hours of the interview taking place, and other members of the research team will be available via phone during scheduled interviews times. The interviews will take place in a secure environment, with the researcher maintaining their own safety at all times with support from nursing staff on the unit, who will know the researcher's whereabouts at all times and having access to a personal duress alarm.

The first author, responsible for data collection, was previously employed by the hospital where recruitment is to take place, therefore women whom she was previously involved with in the delivery of their care, will not be approached to participate in the study. This will be confirmed by the first author, with admission information sought to confirm when required. The previous therapeutic relationship may influence recruitment, creating bias.

\section{Dissemination of information about the study}

Findings of this study will be reported in the first author's doctoral thesis, due for submission in 2023. Results will be published in peer-reviewed journals and presented at local, national and international conferences in this field, as well as reported to the organisation. Where requested, results will also be provided to participants.

\section{Twitter Alison Claire Hansen @AlisonClaireH}

Acknowledgements This research was supported by an Australian Government Research Training Program (RTP) Scholarship. The first author acknowledges the Australian Nurses Memorial Centre who through the award of a Scholarship, helped enable postgraduate nursing studies.

Contributors $\mathrm{ACH}$ is responsible for the original concept of this study. All authors (ACH, MH, RR and $\mathrm{KJI})$ were involved in the study design and development of the protocol. ACH drafted this protocol manuscript with all other authors ( $\mathrm{MH}, \mathrm{RR}$ and $\mathrm{KJI})$ contributing to the content and providing feedback. All authors have read and approved the final manuscript. 
Funding The authors have not declared a specific grant for this research from any funding agency in the public, commercial or not-for-profit sectors.

Competing interests None declared.

Patient and public involvement Patients and/or the public were not involved in the design, or conduct, or reporting, or dissemination plans of this research.

Patient consent for publication Not required.

Provenance and peer review Not commissioned; externally peer reviewed.

Open access This is an open access article distributed in accordance with the Creative Commons Attribution Non Commercial (CC BY-NC 4.0) license, which permits others to distribute, remix, adapt, build upon this work non-commercially, and license their derivative works on different terms, provided the original work is properly cited, appropriate credit is given, any changes made indicated, and the use is non-commercial. See: http://creativecommons.org/licenses/by-nc/4.0/.

ORCID iD

Alison Claire Hansen http://orcid.org/0000-0002-1147-2447

\section{REFERENCES}

1 Australian Institute of Health and Welfare. Mental health services in Australia, 2020. Available: https://www.aihw.gov.au/reports/mentalhealth-services/mental-health-services-in-australia/report-contents/ restrictive-practices/seclusion [Accessed Apr 2020].

2 Melbourne Social Equity Institute. Seclusion and restraint project: report, Melbourne, 2014. Available: https://socialequity.unimelb.edu. au/_data/assets/pdf_file/0017/2004722/Seclusion-and-Restraintreport.PDF

3 Turner KV, Mooney P. A comparison of seclusion rates between intellectual disability and non-intellectual disability services: the effect of gender and diagnosis. J Forens Psychiatry Psychol 2016;27:265-80.

4 Huckshorn KA. Re-designing state mental health policy to prevent the use of seclusion and restraint. Adm Policy Ment Health 2006;33:482-91.

5 Huckshorn KA. Reducing seclusion restraint in mental health use settings: core strategies for prevention. J Psychosoc Nurs Ment Health Serv 2004;42:22-33.

6 Lepping P, Masood B, Flammer E, et al. Comparison of restraint data from four countries. Soc Psychiatry Psychiatr Epidemiol 2016;51:1301-9.

7 Steinert T, Lepping P, Bernhardsgrütter R, et al. Incidence of seclusion and restraint in psychiatric hospitals: a literature review and survey of international trends. Soc Psychiatry Psychiatr Epidemiol 2010;45:889-97.

8 Newton JR, Bosanac P, Copolov D, et al. Targeting zero: implications for public psychiatric services. Aust N Z J Psychiatry 2017;51:560-2.

9 Nicholls TL, Brink J, Greaves C, et al. Forensic psychiatric inpatients and aggression: an exploration of incidence, prevalence, severity, and interventions by gender. Int J Law Psychiatry 2009;32:23-30.

10 de Vogel V, Nicholls TL. Gender matters: an introduction to the special issues on women and girls. Int J Forensic Ment Health 2016;15:1-25.

11 Parkes JH, Freshwater DS. The journey from despair to hope: an exploration of the phenomenon of psychological distress in women residing in British secure mental health services. J Psychiatr Ment Health Nurs 2012;19:618-28.

12 Ahmed A, Lepnurm M. Seclusion practice in a Canadian forensic psychiatric hospital. J Am Acad Psychiatry 2001;29:303-9.

13 Bartlett A, Walker T, Harty MA, et al. Health and social care services for women offenders: current provision and a future model of care. $J$ Forens Psychiatry Psychol 2014:1-11.

14 Bland J, Mezey G, Dolan B. Special women, special needs: a descriptive study of female special hospital patients. The Journal of Forensic Psychiatry 1999;10:34-45.

15 Cooke K, Bailey D. Women's experience of forensic mental health services: implications for practice. J MH Training, Ed and Practice 2011;6:186-94.

16 Meehan T, Vermeer C, Windsor C. Patients' perceptions of seclusion: a qualitative investigation. J Adv Nurs 2000;31:370-7.

17 Creswell JW. Research design: qualitative, quantitative, and mixed methods approaches. 3rd ed. Thousand Oaks, California: SAGE Publications, 2009.

18 Sandelowski M. Whatever happened to qualitative description? Res Nurs Health 2000;23:334-40.

19 Mullen PE. Forensic mental health. Br J Psychiatry 2000;176:307-11.

20 Harris PA, Taylor R, Thielke R, et al. Research electronic data capture (REDCap)--a metadata-driven methodology and workflow process for providing translational research informatics support. J Biomed Inform 2009;42:377-81.

21 Harris PA, Taylor R, Minor BL, et al. The REDCap Consortium: building an international community of software platform partners. $J$ Biomed Inform 2019;95:103208.

22 Vittinghoff E, McCulloch CE. Relaxing the rule of ten events per variable in logistic and COX regression. Am J Epidemiol 2007;165:710-8.

23 Vasileiou K, Barnett J, Thorpe S, et al. Characterising and justifying sample size sufficiency in Interview-Based studies: systematic analysis of qualitative health research over a 15-year period. BMC Med Res Methodol 2018;18.

24 Braun V, Clarke V. (Mis)conceptualising themes, thematic analysis, and other problems with Fugard and Potts' (2015) sample-size tool for thematic analysis. Int J Soc Res Methodol 2016;19:739-43.

25 Braun V, Clarke V. Using thematic analysis in psychology. Qual Res Psychol 2006;3:77-101.

26 Nowell L, White D, Moules N, et al. Thematic analysis: Striving to meet the Trustworthiness criteria. Int J Qual Methods 2017;16:1-13.

27 Hansen A, Hazelton M, Rosina R, et al. Factors associated with seclusion use in forensic mental health settings: an integrative review. Int J Forensic Ment Health 2020;19:198-213.

28 National Health and Medical Research Council. Ethical conduct in research with Aboriginal and Torres Strait Islander peoples and communities: guidelines for researchers and stakeholders, 2018. Available: https://www.nhmrc.gov.au/about-us/resources/ethicalconduct-research-aboriginal-and-torres-strait-islander-peoples-andcommunities

29 von Elm E, Altman DG, Egger M, et al. The strengthening the reporting of observational studies in epidemiology (STROBE) statement: guidelines for reporting observational studies. Ann Intern Med 2007;147:573.

30 Tong A, Sainsbury P, Craig J. Consolidated criteria for reporting qualitative research (COREQ): a 32-item checklist for interviews and focus groups. Int J Qual Health Care 2007;19:349-57.

31 Mental health act (VIC) 2014. 